\title{
Parallaxes and infrared photometry of three Y0 dwarfs
}

\author{
R. L. Smart, ${ }^{1,6 \star} \dagger$ D’aniel Apai, ${ }^{2,3 \star}$ J. Davy Kirkpatrick, ${ }^{4 \star}$ S. K. Leggett, ${ }^{5}$ F. Marocco, ${ }^{6}$ \\ Jane E. Morrison, ${ }^{2}$ H. R. A. Jones, ${ }^{6}$ D. Pinfield, ${ }^{6}$ P. Tremblin ${ }^{7}$ and D. S. Amundsen ${ }^{8}$ \\ ${ }^{1}$ Istituto Nazionale di Astrofisica, Osservatorio Astrofisico di Torino, Strada Osservatorio 20, I-10025 Pino Torinese, Italy \\ ${ }^{2}$ Steward Observatory, University of Arizona, 933 N. Cherry Avenue, Tucson, AZ 85721, USA \\ ${ }^{3}$ Lunar and Planetary Laboratory, 1629 E. University Boulevard, University of Arizona, Tucson, AZ 85721, USA \\ ${ }^{4}$ Infrared Processing and Analysis Center, California Institute of Technology, MS 100-22, Pasadena, CA 91125, USA \\ ${ }^{5}$ Gemini Observatory, 670 N. A'ohoku Place, Hilo, HI 96720, USA \\ ${ }^{6}$ School of Physics, Astronomy and Mathematics, University of Hertfordshire, College Lane, Hatfield AL10 $9 A B$, UK \\ ${ }^{7}$ Maison de la Simulation, CEA-CNRS-INRIA-UPS-UVSQ, USR 3441, Centre détude de Saclay, F-91191 Gif-Sur-Yvette, France \\ ${ }^{8}$ Astrophysics Group, University of Exeter, Exeter EX4 4QL, UK
}

Accepted 2017 March 22. Received 2017 March 13; in original form 2016 September 6

\begin{abstract}
We have followed up the three Y0 dwarfs WISEPA J041022.71+150248.5, WISEPA J173835.53+273258.9 and WISEPC J205628.90+145953.3 using the United Kingdom Infrared Telescope Wide Field Camera. We find parallaxes that are more consistent and accurate than previously published values. We estimate absolute magnitudes in photometric passbands from $Y$ to $W 3$ and find them to be consistent between the three Y0 dwarfs indicating that the inherent cosmic absolute magnitude spread of these objects is small. We examine the Mauna Kea Observatory system $J$ magnitudes over the $4 \mathrm{yr}$ time line and find small but significant monotonic variations. Finally, we estimate physical parameters from a comparison of spectra and parallax to equilibrium and non-equilibrium models finding values consistent with solar metallicity, an effective temperature of 450-475 K and $\log g$ of 4.0-4.5.
\end{abstract}

Key words: techniques: spectroscopic-astrometry-parallaxes - brown dwarfs.

\section{INTRODUCTION}

Y dwarfs represent the coolest collapsed objects outside the Solar system known to date. They exhibit strong methane absorption where the Wide-field Infrared Survey Explorer mission (WISE; Wright et al. 2010) $W 13.4 \mu \mathrm{m}$ filter is centred and emit about half their energy in the WISE W2 $4.6 \mu \mathrm{m}$ passband (Mainzer et al. 2011). This makes the $W 1-W 2$ colour very distinct for these objects and most of the known Y dwarfs have been discovered following their identification as colour-selected candidates in the WISE data (e.g. Cushing et al. 2011).

The temperatures of the Y0 subclass dwarfs are believed to be around $400 \mathrm{~K}$ and their masses to be between 5 and $30 M_{\text {Jup }}$ (Cushing et al. 2011), overlapping in physical parameter space with many exoplanets, so they can be used as surrogates to understand the atmospheric processes of exoplanets. The older examples will hold the chemical imprint of the early Galaxy and the distribution in age may help map out the evolution of formation mechanisms over the Galaxy's lifetime.

In this contribution, we discuss the three objects WISEPA J041022.71+150248.5, WISEPA J173835.53+273258.9 and

^E-mail: smart@oato.inaf.it (RLS); apai@as.arizona.edu (DA); davy@ipac.caltech.edu (DK)

$\dagger$ Leverhulme Visiting Professor.
WISEPC J205628.90+145953.3 which we will refer to as 0410 , 1738 and 2056, respectively. These were all originally presented in Cushing et al. (2011) and classified as spectral types Y0. First we discuss the astrometry, then the photometry and finally we combine these observations with published spectra and models to estimate physical parameters.

\section{ASTROMETRIC ANALYSIS}

\subsection{Observational data}

The astrometric observations were all made on the United Kingdom Infrared Telescope (UKIRT) 3.8-m telescope using the Wide Field Camera (WFCAM), which was the combination used to produce the UKIRT Infrared Deep Sky Survey (UKIDSS, Warren et al. 2007). All observations are carried out in the queue override mode, allowing us to be very flexible in the scheduling, maximizing the parallax factor and observing close to meridian passage. The first observations of these objects were made in 2011 September following a Director Discretionary Time request (U/11B/D1). During the 2012A semester they were included as part of the UKIRT ultracool dwarf parallax program described in Smart et al. (2010) and Marocco et al. (2010, hereafter MSJ10). In 2014, via a request to the University of Arizona (U/14B/UA15) we obtained further observations. The results published here are based on observations from 
Table 1. Parallaxes and proper motions for UKIRT Y0 targets.

\begin{tabular}{|c|c|c|c|c|c|c|c|c|c|}
\hline $\begin{array}{l}\text { Short } \\
\text { name }\end{array}$ & $\begin{array}{c}\text { RA Dec. } \\
\text { (h:m:s), (':':") }\end{array}$ & $\begin{array}{l}\text { Epoch } \\
\text { (yr) }\end{array}$ & $\begin{array}{l}\text { Absolute } \pi \\
\text { (mas) }\end{array}$ & $\begin{array}{c}\mu_{\alpha} \\
\left(\operatorname{mas~yr}^{-1}\right)\end{array}$ & $\begin{array}{c}\mu_{\delta} \\
\left(\operatorname{mas~yr}^{-1}\right)\end{array}$ & $\begin{array}{l}\text { COR } \\
\text { (mas) }\end{array}$ & $N_{*}, N_{\mathrm{e}}$ & $\begin{array}{l}\Delta T \\
(\mathrm{yr})\end{array}$ & $\begin{array}{c}V_{\tan } \\
\left(\mathrm{km} \mathrm{s}^{-1}\right)\end{array}$ \\
\hline 0410 & $4: 10: 23.0,+15: 02: 37.8$ & 2014.9551 & $144.3 \pm 9.9$ & $956.8 \pm 5.6$ & $-2221.2 \pm 5.5$ & 0.97 & 99,19 & 4.36 & $79.4 \pm 5.4$ \\
\hline 1738 & $17: 38: 35.6,+27: 32: 57.8$ & 2013.2584 & $128.5 \pm 6.3$ & $345.0 \pm 5.7$ & $-340.1 \pm 5.1$ & 0.63 & 293,18 & 4.53 & $17.9 \pm 0.9$ \\
\hline 2056 & $20: 56: 29.0,+14: 59: 54.6$ & 2012.8274 & $148.9 \pm 8.2$ & $826.4 \pm 5.5$ & $530.7 \pm 8.5$ & 0.67 & 452,18 & 4.54 & $31.3 \pm 1.7$ \\
\hline
\end{tabular}

$\mathrm{COR}=$ correction to absolute parallax, $N_{*}=$ number of reference stars, $N=$ number of epochs, $\Delta \mathrm{T}=$ epoch range, $V_{\tan }=$ tangential velocity.

September 2011 to April 2016. The basic procedures for observing, image treatment and parallax determination follow those described in MSJ10.

One of the most important aspects in the determination of smallfield parallaxes is stability of the focal plane and repetition of the observational procedure. This is particularly true for the large offaxis detectors of the WFCAM instrument. In the MSJ10 program, we required that the targets are observed in the same physical position on the focal plane as the discovery image in the UKIDSS survey. As these Y0 dwarfs were not in the UKIDSS survey we had the ability to place the target on any of the four chips. For 1738 and 2056, they were placed in the most central quadrant - with respect to the optical axis - of chip 3. This region being close to the optical axis is astrometrically 'quiet'. For 0410, in an attempt to also include the T6 dwarf WISEP J041054.48+141131.6 in chip 1 , we placed it in the top outside quadrant of chip 4. Unfortunately the T6 is not in chip 1 as we hoped, but once the first image was taken we kept the same relative position.

For each target we only used reference stars within a limited radius. The size of the radius is a compromise between limiting the number of reference stars and having a large astrometrically complex area to transform. For all targets we choose 2 arcmin which provided over 50 reference stars. There is a factor of 4 difference between the number of reference stars for 2056 versus 0410 (see $N_{*}$ in Table 1) but 50 was still considered sufficient to astrometrically model such a small area.

As these objects are fainter in the $J$ band than the other targets in the MSJ10 program we increased the exposure time following a 5 jitter (dithered) 3.2 arcsec cross pattern, and at each jitter position we made four exposures in $2 \times 2$ microstepped positions of 1.5 pixels, where each exposure consists of four co-added $10 \mathrm{~s}$ images. The total exposure time is therefore $5 \times 4 \times 4 \times 10=800 \mathrm{~s}$. In average conditions this provides a signal to noise of 50 at Mauna Kea Observatory system (MKO) $J=19.5$.

All observations are reduced using the standard WFCAM Cambridge Astronomical Survey Unit (CASU) pipeline. We transformed all frames to a base frame using a simple six constant linear astrometric fit. We then removed any frames that have an average reference star error larger than the mean error for all frames plus three standard deviations about that mean in either coordinate or have less than 12 stars in common with the base frame. Each observation has a quoted positional error from the UKIRT pipeline based on the profile fitting program and the errors in the transformation parameters. However, there remains a systematic contribution to the error that changes from night to night. For this reason, when fitting for the astrometric parameters to the individual observations on the combined frames, we treated each observation with equal weight and then calculated the final error on the target parameters from the covariance matrix of the solution scaled by the error of unit weight. This fit is also iterated removing any observations where the combined residual in the two coordinates is greater than three times the sigma of the whole solution.
The solutions were tested for robustness using bootstrap-like testing where we iterate through the sequence selecting different frames as the base frame thus making many solutions that incorporate slightly different sets of reference stars and starting from different dates. We select all solutions with (i) a parallax within $1 \sigma$ of the median solution, (ii) the number of included observations in the top 10 per cent and (iii) at least 20 reference stars in common to all frames. From this subset, for this publication, we have selected the one with the smallest error. More than 90 per cent of the solutions are within $1 \sigma$ of the published solution.

To these relative parallaxes we add a correction (COR in Table 1) to find the astrophysically useful absolute parallaxes. The COR is estimated from the average magnitude of the reference stars and the model of Mendez \& van Altena (1996) transformed into the $J$ band.

\subsection{Astrometric parameters}

In Table 1, we report the derived absolute parallaxes, proper motions and details of the solutions for the UKIRT sequences. In Fig. 1, we plot the observations and the predicted movement of the targets from our parameters. The result for 0410 is lower precision than 1738 and 2056 probably due to the higher proper motion, since any error in our estimation of this motion will propagate into the parallax estimate.

\subsection{Comparison to published values}

Parallaxes for these objects have been measured by three teams Marsh et al. (2013), Beichman et al. (2014) and Dupuy \& Kraus (2013, hereafter D\&K13). Marsh et al. (2013) and Beichman et al. (2014) used combinations of WISE W2, WIRC $J$, NEWFIRM, Spitzer channel 2 and HST $J$ observations with a significant overlap of the two sets of observations. The Marsh et al. (2013) results for these targets were based on 7-9 observations while those of the Beichman et al. (2014), coming later, were based on 14-16. The results for D\&K13 were based on 5 Spitzer channel 1 observations. In Kirkpatrick et al. (2011), they also provide parallactic distances but they were preliminary estimates from the Marsh et al. (2013) work so we have only reported the latter values. Considering all the published targets there are 9 Y0 dwarfs with more than one estimated parallax including the three objects under study here. In Table 2, we have reported all results in common between the three cited works and the results from this contribution.

In Fig. 2, we plot the differences of the parallax values for the nine targets with respect to the D\&K13 value divided by the error of the two estimates combined in quadrature. The D\&K13 values are relative but the difference between relative and absolute parallax is negligible compared to the random errors. The 1541 and 1738 Marsh et al. (2013) values are very different from the other values for these targets and are also significantly lower than the predicted spectroscopic parallax so we assume these are compromised. Given the short-time baseline and mixed-observational sources for the 


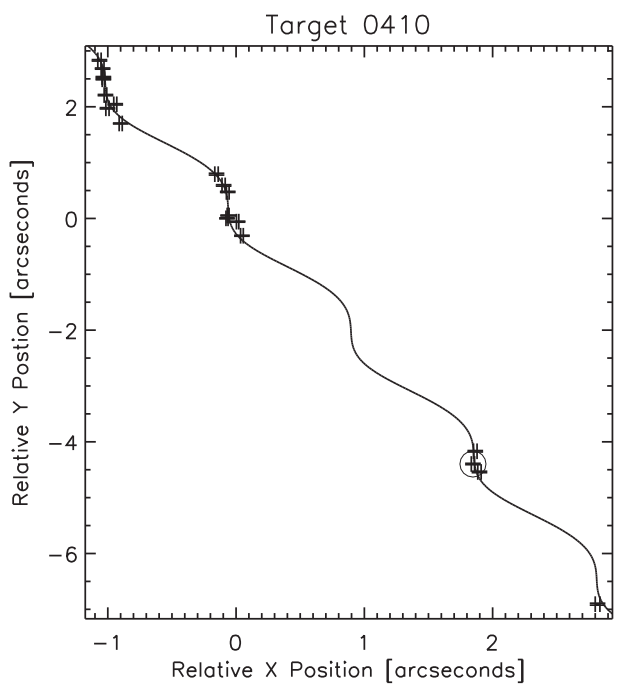

Table 2. Comparison of parallaxes and proper motions for $\mathrm{Y} 0$ dwarfs in different programs.

\begin{tabular}{|c|c|c|c|c|}
\hline $\begin{array}{l}\text { Short } \\
\text { name }\end{array}$ & $\begin{array}{l}\mu_{\alpha} \\
\left(\operatorname{mas~yr}^{-1}\right)\end{array}$ & $\begin{array}{l}\mu_{\delta} \\
\left(\operatorname{mas} \mathrm{yr}^{-1}\right)\end{array}$ & $\begin{array}{l}\text { Absolute } \pi \\
\text { (mas) }\end{array}$ & Ref \\
\hline 0254 & $2588 \pm 27$ & $273 \pm 27$ & $135 \pm 15$ & 2 \\
\hline 0254 & $2578 \pm 42$ & $309 \pm 50$ & $185 \pm 42$ & 3 \\
\hline 0410 & $966 \pm 13$ & $-2218 \pm 13$ & $160 \pm 9$ & 1 \\
\hline 0410 & $958 \pm 37$ & $-2229 \pm 29$ & $132 \pm 15$ & 2 \\
\hline 0410 & $974 \pm 79$ & $-2144 \pm 72$ & $233 \pm 56$ & 3 \\
\hline 0410 & $956 \pm 06$ & $-2223 \pm 6$ & $144 \pm 10$ & 4 \\
\hline 1405 & $-2263 \pm 47$ & $288 \pm 41$ & $129 \pm 19$ & 2 \\
\hline 1405 & $-2297 \pm 96$ & $212 \pm 137$ & $133 \pm 81$ & 3 \\
\hline 1541 & $-857 \pm 12$ & $-087 \pm 13$ & $176 \pm 9$ & 1 \\
\hline 1541 & $-870 \pm 130$ & $-013 \pm 58$ & $74 \pm 31$ & 2 \\
\hline 1541 & $-983 \pm 111$ & $-276 \pm 116$ & $-21 \pm 94$ & 3 \\
\hline 1738 & $317 \pm 09$ & $-321 \pm 11$ & $128 \pm 10$ & 1 \\
\hline 1738 & $292 \pm 63$ & $-396 \pm 22$ & $102 \pm 18$ & 2 \\
\hline 1738 & $348 \pm 71$ & $-354 \pm 55$ & $66 \pm 50$ & 3 \\
\hline 1738 & $346 \pm 6$ & $-338 \pm 5$ & $129 \pm 6$ & 4 \\
\hline 1741 & $-509 \pm 35$ & $-1463 \pm 32$ & $180 \pm 15$ & 2 \\
\hline 1741 & $-495 \pm 11$ & $-1472 \pm 13$ & $176 \pm 26$ & 3 \\
\hline 1804 & $-269 \pm 10$ & $035 \pm 11$ & $80 \pm 10$ & 1 \\
\hline 1804 & $-242 \pm 26$ & $017 \pm 22$ & $60 \pm 11$ & 2 \\
\hline 1828 & $1024 \pm 7$ & $174 \pm 6$ & $106 \pm 7$ & 1 \\
\hline 1828 & $1020 \pm 15$ & $173 \pm 16$ & $70 \pm 14$ & 2 \\
\hline 2056 & $812 \pm 9$ & $534 \pm 8$ & $140 \pm 9$ & 1 \\
\hline 2056 & $761 \pm 46$ & $500 \pm 21$ & $144 \pm 23$ & 2 \\
\hline 2056 & $881 \pm 57$ & $544 \pm 42$ & $144 \pm 44$ & 3 \\
\hline 2056 & $828 \pm 6$ & $532 \pm 8$ & $149 \pm 8$ & 4 \\
\hline
\end{tabular}

References: 1, Beichman et al. (2014); 2, Dupuy \& Kraus (2013); 3, Marsh et al. (2013); 4, this work. Note D\&K13 only published relative parallaxes which is what we have reported here. The targets are 0254: WISE J025409.45+022359.1; 0410: WISEPA J041022.71+150248.5; 1405 : WISE J140518.40+553421.5; 1541: WISE J154151.65-225025.2; 1738 : WISE J173835.52+273258.9; 1741: WISE J174124.26+255319.5; 1804: WISE J180435.40+311706.1; 1828: WISE J182831.08+265037.8; 2056 ; WISE J205628.90+145953.3.

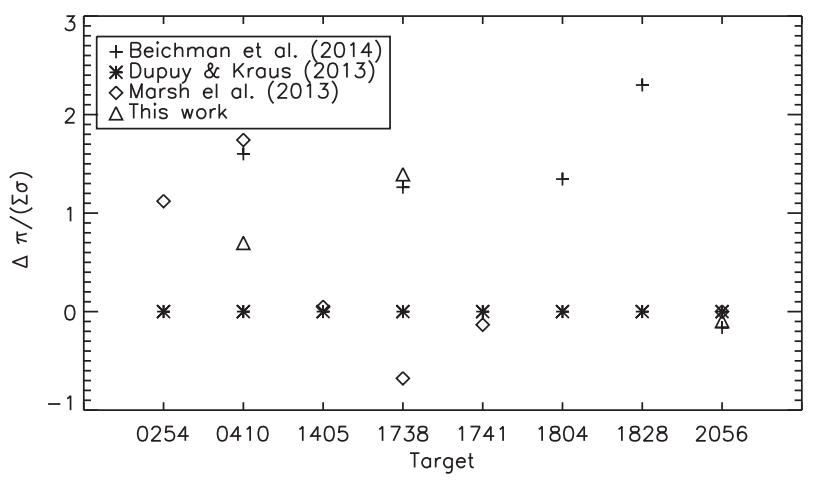

Figure 2. Parallaxes differences compared to the D\&K13 values as multiples of the combined standard deviation for each solution. The D\&K13 value is used as the reference as they have published a parallaxe for all of the Y0 dwarfs considered here. References and target abbreviations as in Table 2. So, for example the Marsh et al. (2013) value of 0254 is one combined sigma larger than the D\&K13 value. 
Marsh et al. (2013) work, it is to be expected that in some solutions the proper motion and the parallax were not disentangled correctly. Apart from the two low Marsh et al. (2013) values, all the other D\&K13 estimates appear as underestimates, on average by one combined sigma.

D\&K13 published relative parallaxes not absolute ones because they felt the correction was negligible. The corrections we have applied are less than 1 mas and since the average reference star is fainter in the Spitzer fields we would expect the D\&K13 corrections to be even smaller. A correction will reduce the difference in the right direction but we agree with the authors that it cannot be the main cause of the observed difference. We also note in Tinney et al. (2014) they also find that the D\&K13 parallax estimates are low for other targets. This comparison to the D\&K13 values indicates that the most reliable results are those of Beichman et al. (2014). The difference between the results published here and those of Beichman et al. (2014) are all within $1 \sigma$. We consider this a confirmation of our procedures, parallax estimates and, more importantly, error estimates.

\subsection{Search for common proper motion objects and moving group membership}

We searched for common proper motion companions to our three $\mathrm{Y}$ dwarfs within the Hipparcos Main Catalogue, the Gliese Catalogue of Nearby Stars, the Tycho- 2 catalogue and the Fourth U.S. Naval Observatory CCD Astrograph Catalogue. We looked for objects with differences in both proper motion components $<3 \sigma$ and a maximum projected separation $<100000$ au. The search returned no matches.

We used the BANYAN II online tool ${ }^{1}$ (Malo et al. 2013; Gagné et al. 2014) to assess the membership of our targets to the nearby moving groups. None of the targets have significant probability of belonging to any of the moving groups. However, for 2056, we obtained a probability of 44 percent to be an old field member and 56 percent to be a young field member, suggesting that this object might pertain to a slightly younger population. The tangential velocity, listed in the last column of Table 1, of 0410 is significantly larger than both 1738 and 2056 suggesting it might be old but it does not exceed the $V_{\tan }>100 \mathrm{~km} \mathrm{~s}^{-1}$ criteria adopted for ultracool dwarfs belonging to either the Galactic thick disc or halo (Faherty et al. 2009).

We also used LACEwING ${ }^{2}$ to access membership assuming that the three targets are field objects - e.g. that we have no evidence of youth. Again none of the targets show a significant probability of being in a moving group though 2056 did have a 30 percent probability of belonging to the Argus group. We conclude that these objects are not members of any known moving groups and are probably just local field members.

\section{PHOTOMETRIC ANALYSIS}

\subsection{Photometric analysis of WFCAM data}

The CASU pipeline estimates the MKO $J$ magnitude of our targets using 100-200 calibrating stars from the Two Micron All Sky Survey (hereafter 2MASS; Skrutskie et al. 2006) as described in Hodgkin et al. (2009). The systematic errors of the calibration from

\footnotetext{
${ }^{1}$ http://www.astro.umontreal.ca/g̃agne/banyanII.php

${ }^{2}$ https://github.com/ariedel/lacewing
}
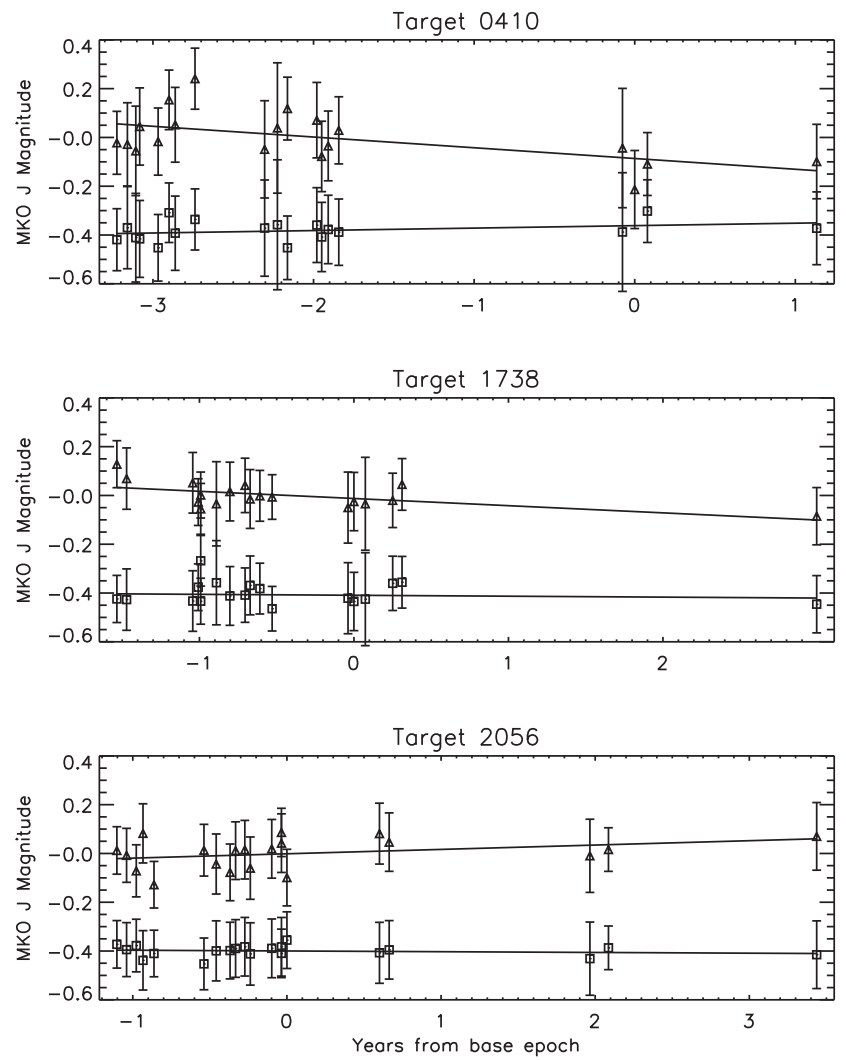

Figure 3. MKO $J$ magnitude variations as a function of time for 0410, 1738 and 2056, respectively. In each plot, we have included an anonymous object that is nearby in position and magnitude and plotted its magnitude variation offset by $-0.4 \mathrm{mag}$.

Table 3. Mean MKO $J$ magnitudes and variations with time.

\begin{tabular}{lccccr}
\hline Target & $N$ & $\begin{array}{c}\langle\mathrm{J}\rangle \\
(\mathrm{mag})\end{array}$ & $\begin{array}{c}\sigma \\
(\mathrm{mag})\end{array}$ & $\begin{array}{c}\sigma_{\text {mean }} \\
(\mathrm{mag})\end{array}$ & \multicolumn{1}{c}{$\begin{array}{c}\text { Slope } \\
\left(\mathrm{mag} \mathrm{yr}^{-1}\right)\end{array}$} \\
\hline 0410 & 19 & 19.137 & 0.103 & 0.031 & $-0.044 \pm 0.016$ \\
Anon & & 18.789 & 0.219 & 0.054 & $0.010 \pm 0.038$ \\
1738 & 18 & 19.539 & 0.051 & 0.023 & $-0.029 \pm 0.010$ \\
Anon & & 19.048 & 0.068 & 0.026 & $-0.004 \pm 0.058$ \\
2056 & 20 & 19.237 & 0.062 & 0.024 & $0.018 \pm 0.012$ \\
Anon & & 18.593 & 0.005 & 0.020 & $-0.003 \pm 0.005$ \\
\hline
\end{tabular}

The 'Anon' entry for each target is the straight line fit to the anonymous field star in each sequence. The slope is always smaller and within $1 \sigma$ zero.

2MASS stars are estimated to be better than 1.5 per cent (Hodgkin et al. 2009). The random error is calculated in the pipeline procedures. Following the recommendation in Dye et al. (2006), we have adopted the aperMag3 parameter as the best estimate of the total magnitude for point sources.

In Fig. 3, we plot the variation of the $J$ magnitude for the parallax program observations with respect to the mid-epoch. In Table 3, we report the number of observations, the magnitude, standard deviation, the error of the mean and the slope of the best-fitting straight line. The long-term changes of 0410 and 1738 do appear to be significant, indicating a slow dimming over the period observed and the number of observations is, however, very low and quite noisy. Further observations of these targets are needed to confirm the observed trends.

We adopt the mean magnitude from Table 3 as the best estimate of the MKO magnitude for these targets. To provide a conservative 
Table 4. Published MKO $J$ and $H$ magnitudes and weighted means.

\begin{tabular}{lccl}
\hline Target & MKO J, $\sigma$ & MKO $H, \sigma$ & \multicolumn{1}{c}{ Source } \\
\hline 0410 & $19.44,0.03$ & $20.02,0.05$ & Leggett et al. (2015) \\
& $19.33,0.02$ & $19.90,0.04$ & Schneider et al. (2015) \\
& $19.24,0.05$ & $19.05,0.09$ & Kirkpatrick et al. (2012) \\
& $19.23,0.05$ & - & Mean from Table 3 \\
& $19.34,0.02$ & $19.85,0.03$ & Weighted mean \\
1738 & $19.63,0.05$ & $20.24,0.08$ & Leggett et al. (2015) \\
& $19.55,0.02$ & $20.25,0.03$ & Schneider et al. (2015) \\
& $19.51,0.08$ & $20.39,0.33$ & Kirkpatrick et al. (2012) \\
& $19.52,0.03$ & - & Mean from Table 3 \\
& $19.55,0.02$ & $20.25,0.03$ & Weighted mean \\
2056 & $19.43,0.04$ & $19.96,0.04$ & Leggett et al. (2015) \\
& $19.13,0.02$ & $19.64,0.03$ & Schneider et al. (2015) \\
& $19.23,0.13$ & $19.62,0.31$ & Kirkpatrick et al. (2012) \\
& $19.23,0.03$ & - & Mean from Table 3 \\
& $19.20,0.02$ & $19.75,0.02$ & Weighted mean \\
\hline
\end{tabular}

estimate of the error of the mean, we simply add 0.02 magnitudes to account for the 1.5 per cent systematic error estimates from Hodgkin et al. (2009).

\subsection{Literature photometry}

There are three published MKO $J$ and $H$ values from Leggett et al. (2015), Schneider et al. (2015) and Kirkpatrick et al. (2012) for these targets as reported in Table 4 along with the estimates found in the last section. The differences are significant, reaching $1 \mathrm{mag}$ for the $H$ band of target 0410 . The Leggett et al. (2015) values are consistently fainter than all these values and this was discussed in that paper. Since the number of estimates is too small to allow any meaningful statistical tests, we simply calculate a weighted mean of all values as reported in Table 4 and use this in our further analysis.

In Table 5 along with the weighted-mean $J$ and $H$ magnitudes, we report all published photometry from other bands. From the apparent magnitudes we estimate absolute magnitudes assuming a distance given by the parallax in Table 1 . The error on the distance is the largest contributor to the error in the absolute magnitude. In the

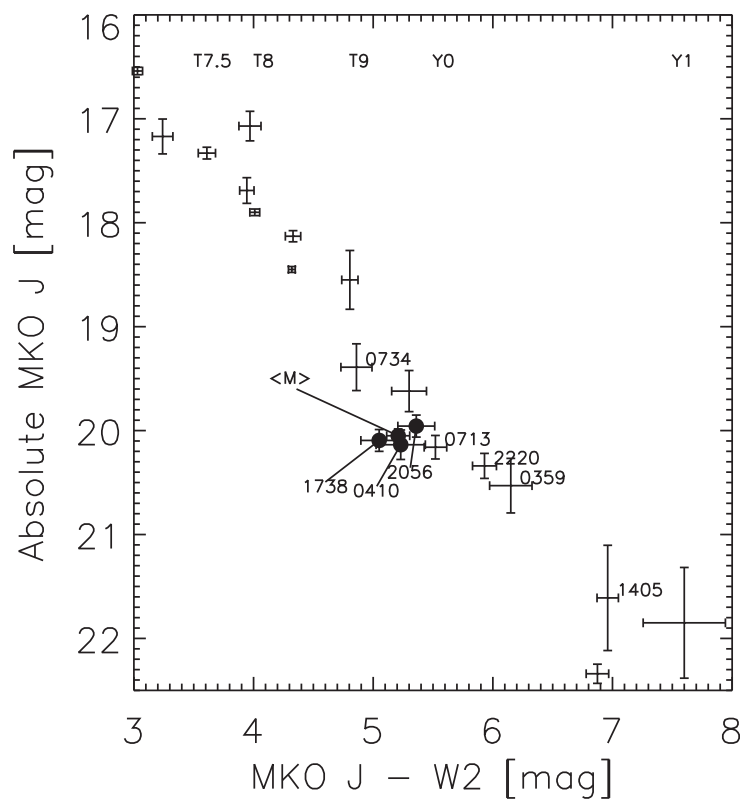

Figure 4. The absolute MKO $J$ magnitude versus the MKO $J-\mathrm{W} 2$ colour for published $>\mathrm{T} 7$ objects from Leggett et al. (2015) along with the objects presented here. The spectral types at the top of the graph are provided just as indicative ranges; the labelled objects are the Y0 dwarfs 0359: WISE J035934.06-540154.6; 0713: WISE J071322.55-291751.9; 0734: WISE J073444.02-715744.0; 1405: WISEP J140518.40+553421.5; 2220: WISE J222055.31-362817.4. The $\langle\mathrm{M}\rangle$ point is the weighted-mean absolute magnitude as found in Table 5 .

last line of Table 5 we include a weighted-mean absolute magnitude and the error of that mean.

In Fig. 4, we plot the absolute MKO $J$ magnitudes versus MKO $J-\mathrm{W} 2$ colour for various $>\mathrm{T} 7$ objects with distances and magnitudes taken from Leggett et al. (2015). The three targets presented here are labelled, as are the other Y0 objects in the sample. The weighted-mean absolute $J$ and $W 2$ magnitudes, $20.05 \pm 0.07$ and $14.84 \pm 0.07$, from Table 5 can be directly compared to the median absolute magnitudes based on $11 \mathrm{Y} 0$ dwarfs with measured distances in Tinney et al. (2014) of $20.32 \pm 1.25$ and $14.65 \pm 0.35$. The difference is large but within $1 \sigma$, and as can be seen from

Table 5. Apparent magnitudes from various sources and mean absolute magnitudes as a function of filter.

\begin{tabular}{|c|c|c|c|c|c|c|c|}
\hline $\begin{array}{l}\text { Band } / \lambda_{\text {eff }} \\
\text { target }\end{array}$ & $\begin{array}{l}z^{1}, \sigma \\
9535\end{array}$ & $\begin{array}{c}\text { MKO } Y^{2}, \sigma \\
10289\end{array}$ & $\begin{array}{l}\text { MKO } J^{3}, \sigma \\
\quad 12444\end{array}$ & $\begin{array}{l}\text { MKO } H^{3}, \sigma \\
\quad 16221\end{array}$ & $\begin{array}{l}\text { MKO } K^{2}, \sigma \\
\quad 21900\end{array}$ & $\begin{array}{l}\text { Spitzer } \operatorname{ch} 1^{4}, \sigma \\
\quad 35075\end{array}$ & $\begin{array}{c}\text { Spitzer } \operatorname{ch} 2^{4}, \sigma \\
44366\end{array}$ \\
\hline 0410 & $22.66,0.09$ & $19.61,0.04$ & $19.34,0.02$ & $19.85,0.03$ & $19.91,0.07$ & $16.64,0.04$ & $14.17,0.02$ \\
\hline 1738 & $22.80,0.09$ & $19.86,0.07$ & $19.55,0.02$ & $20.25,0.03$ & $20.58,0.10$ & $17.09,0.05$ & $14.47,0.02$ \\
\hline 2056 & $23.09,0.08$ & $19.77,0.05$ & $19.20,0.02$ & $19.75,0.02$ & $20.01,0.06$ & $16.03,0.03$ & $13.92,0.02$ \\
\hline$\langle\mathrm{M}\rangle$ & $23.57 \pm 0.08$ & $20.45 \pm 0.07$ & $20.05 \pm 0.07$ & $20.65 \pm 0.07$ & $20.86 \pm 0.08$ & $17.24 \pm 0.07$ & $14.87 \pm 0.07$ \\
\hline Band $/ \lambda_{\text {eff }}$ & WISE $W 1^{4}, \sigma$ & WISE $W 2^{4}, \sigma$ & WISE $W 3^{4}, \sigma$ & $F 125 W^{5}, \sigma$ & $F 140 W^{5}, \sigma$ & Distance & \\
\hline Target & 33526 & 46028 & 115608 & 12305 & 13645 & Modulus & \\
\hline 0410 & $>18.170$ & $14.11,0.05$ & $12.31,0.50$ & $20.00,0.03$ & $19.64,0.02$ & $0.80_{-0.14}^{+0.14}$ & \\
\hline 1738 & $17.71,0.16$ & $14.50,0.04$ & $12.45,0.40$ & $20.22,0.02$ & $19.92,0.02$ & $0.54_{-0.10}^{+0.11}$ & \\
\hline 2056 & $16.48,0.08$ & $13.84,0.04$ & $11.73,0.25$ & $19.81,0.02$ & $19.48,0.02$ & $0.76_{-0.11}^{+0.10}$ & \\
\hline$\langle\mathrm{M}\rangle$ & $17.57 \pm 0.11$ & $14.84 \pm 0.07$ & $12.71 \pm 0.21$ & $20.69 \pm 0.07$ & $20.37 \pm 0.07$ & & \\
\hline
\end{tabular}

References: 1, Lodieu, Béjar \& Rebolo (2013); 2, Leggett et al. (2015); 3, This work; 4, Kirkpatrick et al. (2012); 5, Schneider et al. (2015). The $\lambda_{\text {eff }}$ row is the simple median effective wavelength for a convolution of the nominal filter profile and a Vega spectrum; this is often not appropriate for these objects because of the structure in the underlying spectra but it is provided for reference. The published apparent magnitudes are converted to absolute magnitudes using the distance modulus from the parallaxes presented in Table 1. The $\langle\mathbf{M}\rangle$ row is the weighted-mean absolute magnitude for all three targets along with the error of the mean for each filter. 
Table 6. The best-fitting parameters obtained with our model fitting, compared with those obtained by Cushing et al. (2011). For each target, the first line presents the parameters obtained normalizing both model and target spectrum in the $J$ band, while the second line presents the parameters obtained scaling the observed spectrum to match the measured absolute $J$ magnitude. The metallicity, [M/H], was a fitted parameter for the Tremblin et al. (2015) models, but always came out to be solar for all sets of parameters.

\begin{tabular}{|c|c|c|c|c|c|c|c|c|c|c|c|c|}
\hline \multirow{2}{*}{$\begin{array}{l}\text { Models } \\
\text { Short } \\
\text { name }\end{array}$} & \multicolumn{3}{|c|}{ Tremblin et al. (2015) } & \multicolumn{3}{|c|}{$\begin{array}{c}\text { This paper } \\
\text { Morley et al. (2012) }\end{array}$} & \multicolumn{3}{|c|}{ Morley et al. (2014) } & \multicolumn{3}{|c|}{$\begin{array}{c}\text { Cushing et al. (2011) } \\
\text { Saumon \& Marley (2008) }\end{array}$} \\
\hline & $\begin{array}{l}T_{\text {eff }} \\
(\mathrm{K})\end{array}$ & $\log g$ & $\begin{array}{l}\log \\
K_{\text {zz }}\end{array}$ & $\begin{array}{l}T_{\text {eff }} \\
(\mathrm{K})\end{array}$ & $\log g$ & $f_{\text {sed }}$ & $\begin{array}{l}T_{\text {eff }} \\
(\mathrm{K})\end{array}$ & $\log g$ & $f_{\text {sed }}$ & $\begin{array}{l}T_{\text {eff }} \\
(\mathrm{K})\end{array}$ & $\log g$ & $\begin{array}{l}\log \\
K_{\text {zz }}\end{array}$ \\
\hline \multirow[t]{2}{*}{0410} & 500 & $4.0-4.5$ & 6 & $400-450$ & $4.0-5.0$ & $4-5$ & $350-400$ & $4.5-5.0$ & 5 & 450 & 3.75 & 0 \\
\hline & 450 & $4.0-4.5$ & 6 & 450 & $4.0-4.5$ & $3-5$ & $375-400$ & 4.0 & $5-7$ & & & \\
\hline \multirow[t]{2}{*}{1738} & $475-500$ & $4.0-4.5$ & 6 & 400 & $4.5-5.0$ & $4-5$ & 400 & 5.0 & 5 & 350 & 4.75 & 4 \\
\hline & 475 & 4.0 & 6 & 450 & 4.5 & 5 & 450 & 5.0 & 5 & & & \\
\hline \multirow[t]{2}{*}{2056} & $450-500$ & $4.0-4.5$ & 6 & $400-450$ & $4.5-5.0$ & $4-5$ & $375-400$ & 5.0 & 5 & 350 & 4.75 & 4 \\
\hline & 450 & 4.0 & 6 & 450 & 4.5 & $3-4$ & $400-450$ & 5.0 & 5 & & & \\
\hline
\end{tabular}

Fig. 4 while the spread of Y0 dwarfs appears large the three Y0 dwarfs studied here are however very similar. Despite the systematic differences in parallaxes noted in Section 2.3 the mean absolute magnitudes per spectral type derived by D\&K13 (table S3) for the $Y, J, H, K$, Spitzer channel 1 and 2 bands are consistent at the $1 \sigma$ level with our values.

\section{SPECTROSCOPIC ANALYSIS AND COMPARISON TO MODELS}

We fit the spectra from Cushing et al. (2011) of our targets with the atmospheric models presented in Tremblin et al. (2015) and Morley et al. (2012, 2014). The Tremblin et al. (2015) model grid covers the $200 \mathrm{~K}<T_{\text {eff }}<1000 \mathrm{~K}$ range with $\log g$ values of $4.0,4.5$ and 4.8. We examined both solar metallicity models and metal-poor models with $[\mathrm{M} / \mathrm{H}]=-0.5$ to -0.8 , and both equilibrium and nonequilibrium models with $\log K_{\mathrm{zz}}=6$. The Tremblin et al. (2015) models do not take into account clouds. The models from Morley et al. (2012) are computed for $400 \mathrm{~K} \leq T_{\text {eff }} \leq 1300 \mathrm{~K}, \log g 4.0,4.5$, 5.0 and 5.5, and $2 \leq f_{\text {sed }} \leq 5$. The models from Morley et al. (2014) cover the $200 \mathrm{~K} \leq T_{\text {eff }} \leq 450 \mathrm{~K}$ range, $3.0 \leq \operatorname{logg} \leq 5.0$ and $3 \leq$ $f_{\text {sed }} \leq 7$. The Morley et al. $(2012,2014)$ models assume a patchy cloud coverage with only 50 per cent of the object being covered by clouds.

The Cushing et al. (2011) spectra cover the wavelength range $1-2.4 \mu \mathrm{m}$ at a resolution of $R \approx 300$ for $0410,1.07-1.70 \mu \mathrm{m}$ wavelength range at a low resolving power of $R \approx 130$ for 1738 , and $1.143-1.375 \mu \mathrm{m}$ and $1.431-1.808 \mu \mathrm{m}$ at a resolution or $R \approx$ 2500 for 2056.

We derived the best-fitting parameters by fitting our model grid to the observed spectra using a standard reduced $\chi^{2}$ minimization. We used two slightly different approaches. One normalizes both target and model spectra at the peak of the $J$ band (i.e. $1.26 \mu \mathrm{m}$ ). The other approach makes use of the parallaxes derived here. The Tremblin et al. (2015) models provide flux at $10 \mathrm{pc}$ (assuming a radius of $0.1 \mathrm{R}_{\odot}$ ), which we have re-scaled for the appropriate radius taken from Saumon \& Marley (2008). We then scaled the observed spectra to match the absolute MKO $J$ magnitude, calculated using their measured parallax. A similar procedure was applied to the models from Morley et al. (2012, 2014). To assess the quality of the bestfitting parameters, we adopted the approach described in Cushing et al. (2011). Briefly, we generate 10000 'mimic spectra' for each object by adding Gaussian noise to the observed spectra, preserving the signal-to-noise ratio. Then, we fit these 10000 mimics with the atmospheric models to determine their best-fitting parameters. We adopt the range of parameters encompassed by the standard deviation of the resulting distribution as our best-fitting values.

The results are summarized in Table 6 . The differences between the best-fitting parameters derived with the two methods and the different models are small, but with the Tremblin et al. (2015) models give systematically lower $\log g$ and higher $T_{\text {eff }}$ values than the Morley et al. $(2012,2014)$ models. The reason for this systematic difference is beyond the scope of this contribution but it is probably due to the use of different opacity tables, e.g. Tremblin et al. (2015) uses molecular line lists for ammonia from Yurchenko \& Tennyson (2014) while Morley et al. (2012, 2014) uses those from Yurchenko, Barber \& Tennyson (2011).

For 0410, the best-fitting parameters when normalizing the spectra are $T_{\text {eff }} \sim 450 \mathrm{~K}, \log g \sim 4.5$ and solar metallicity. The only notable exception are the Morley et al. (2014) models that would predict a lower $T_{\text {eff }}$ of 350-400 K. When using the measured parallax we obtain a slightly lower $T_{\text {eff }}$. The spectrum of 0410 and the best-fitting models are plotted in Fig. 5. We note that $500 \mathrm{~K}$ is a higher temperature than previously found for Y0s (e.g. Kirkpatrick et al. 2012; Leggett et al. 2013; Beichman et al. 2014), while the value obtained using the parallax is more in line with the published estimates.

For 1738, the best-fitting parameters given by the various models tend to be more discordant. When normalizing the spectra, the Tremblin et al. (2015) models give $T_{\text {eff }}=500 \mathrm{~K}$ and $\log g=4.5$ (with solar metallicity and $\log K_{\mathrm{zz}}=6$ ). The other models predict a lower $T_{\text {eff }}$ of $400 \mathrm{~K}$ with a higher $\log g$ of $\approx 4.75$. Using our measured parallax mitigates the discrepancy, with the Morley et al. (2012, 2014) models returning best-fitting $T_{\text {eff }}=450 \mathrm{~K}$ and $\log$ $g=4.5-5.0$, closer to the Tremblin et al. values. The spectrum of 1738 and the best-fitting models are plotted in Fig. 6.

For 2056 we have similar situation, with some discrepancy between the best-fitting parameters derived from the different models when normalizing the spectra. The Tremblin et al. (2015) models predict slightly higher $T_{\text {eff }}$ compared to the other models (450$500 \mathrm{~K}$ versus $375-450 \mathrm{~K}$ ), and also in this case the discrepancy is removed when using the measured parallax. The spectrum of 2056 and the best-fitting models are plotted in Fig. 7.

\section{DISCUSSION}

We have found parallaxes with relative errors less than 7 per cent using observations from just one telescope and detector 
Tremblin et al. (2015) models.
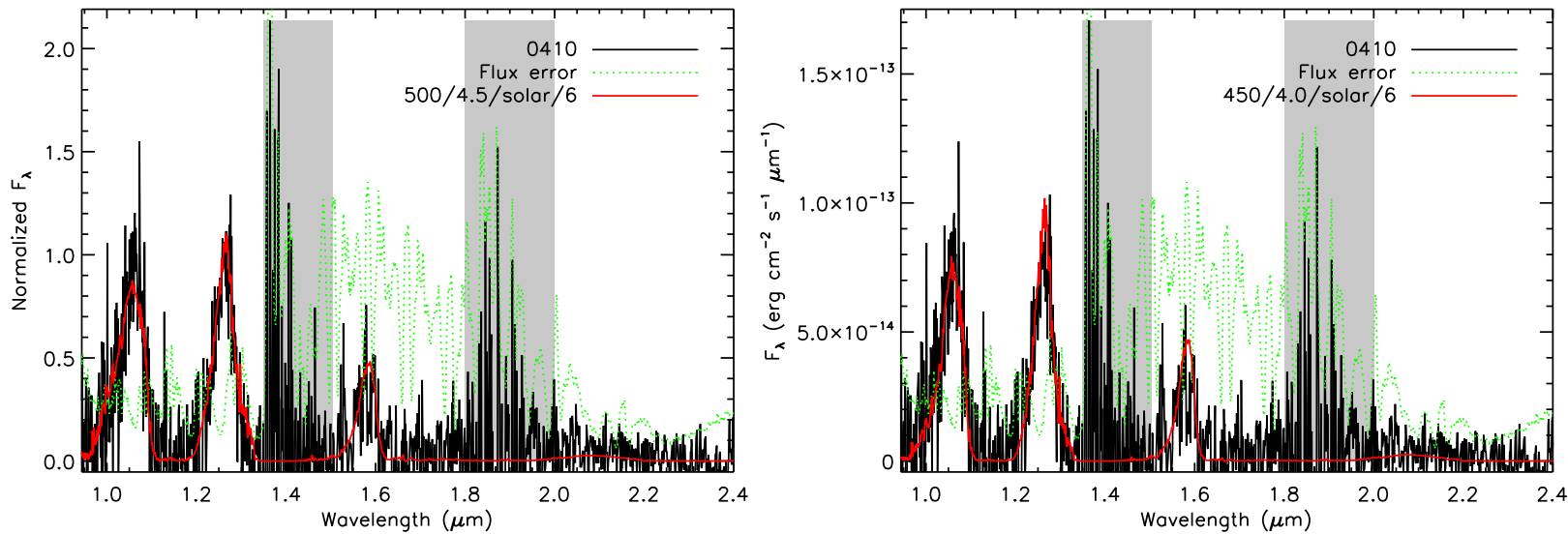

Morley et al. (2012) models.
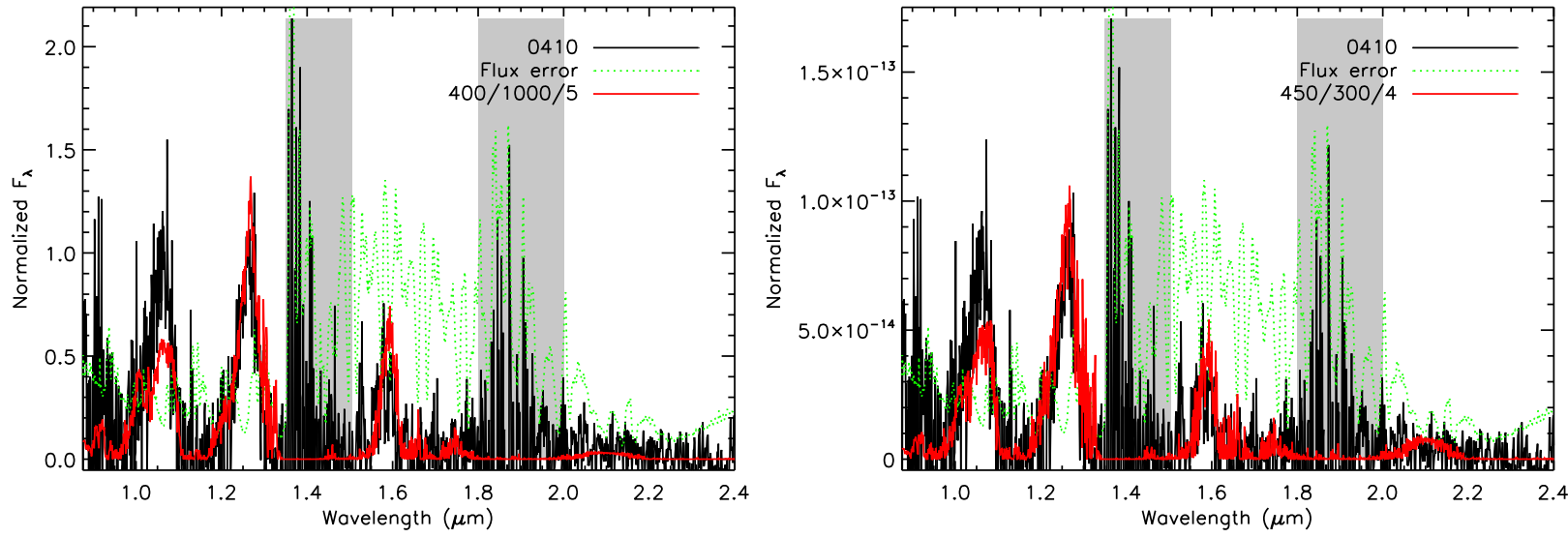

Morley et al. (2014) models.
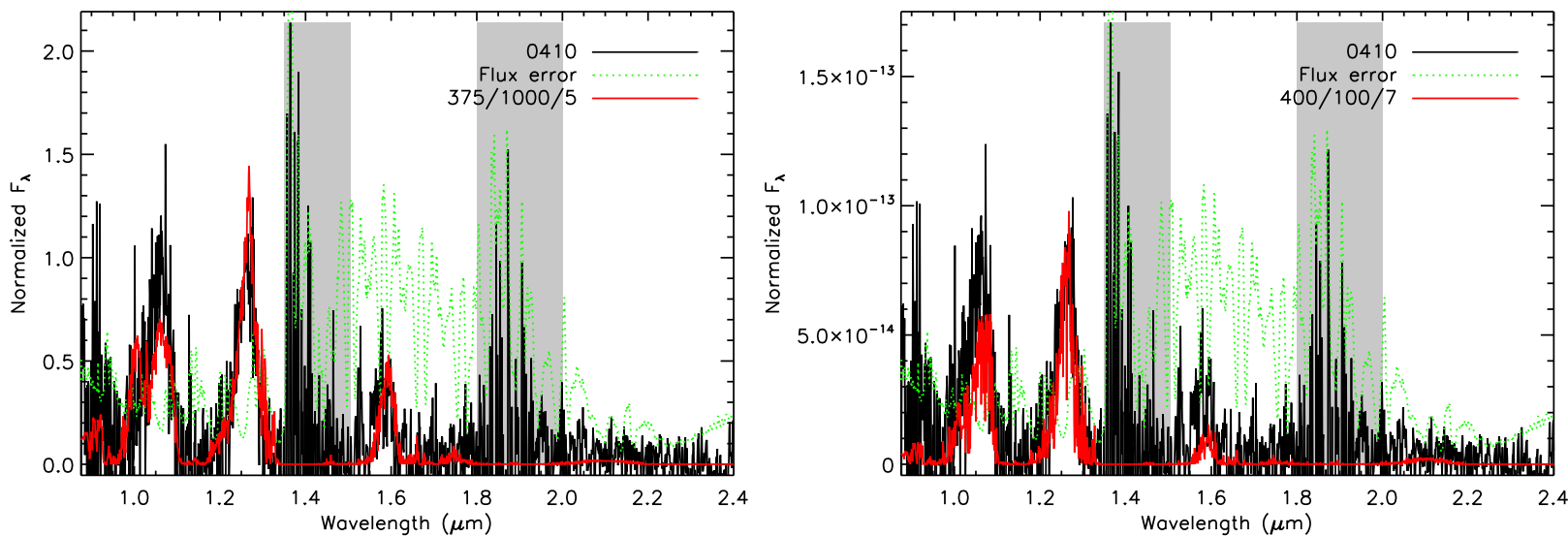

Figure 5. The best-fitting model for 0410, obtained normalizing both model and target spectrum in the $J$ band (left panel) or scaling the observed spectrum to match the measured absolute $J$ magnitude (right panel). The spectrum of 0410 is plotted in black, its associated $1 \sigma$ uncertainty is plotted in green, and the best-fitting model is plotted in red. The shaded area are regions that are heavily effected by telluric absorption so are excluded from the model fitting. The best-fitting parameters are summarized in the legend, following the scheme $T_{\text {eff }} / \log g /[\mathrm{M} / \mathrm{H}] / \log K_{\mathrm{zz}}$ for the Tremblin et al. (2015) models and $T_{\text {eff }} / \mathrm{g} / f_{\text {sed }}$ for the Morley et al. (2012) and Morley et al. (2014) models. 
Tremblin et al. (2015) models.
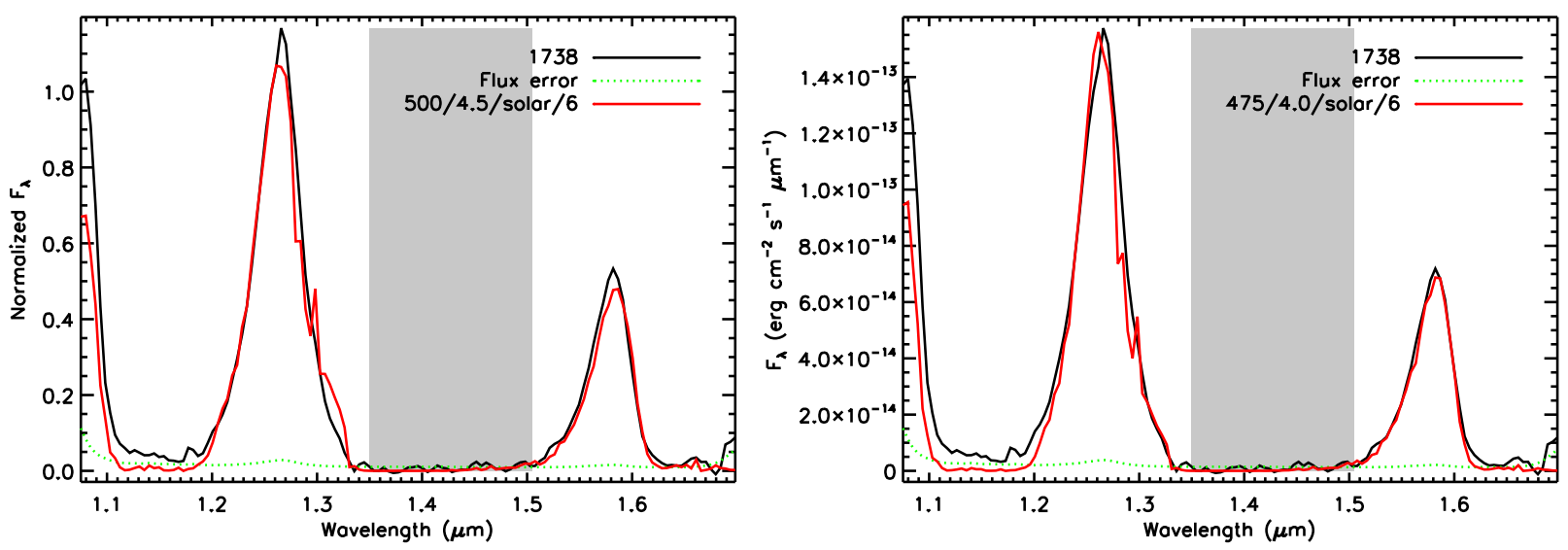

Morley et al. (2012) models.
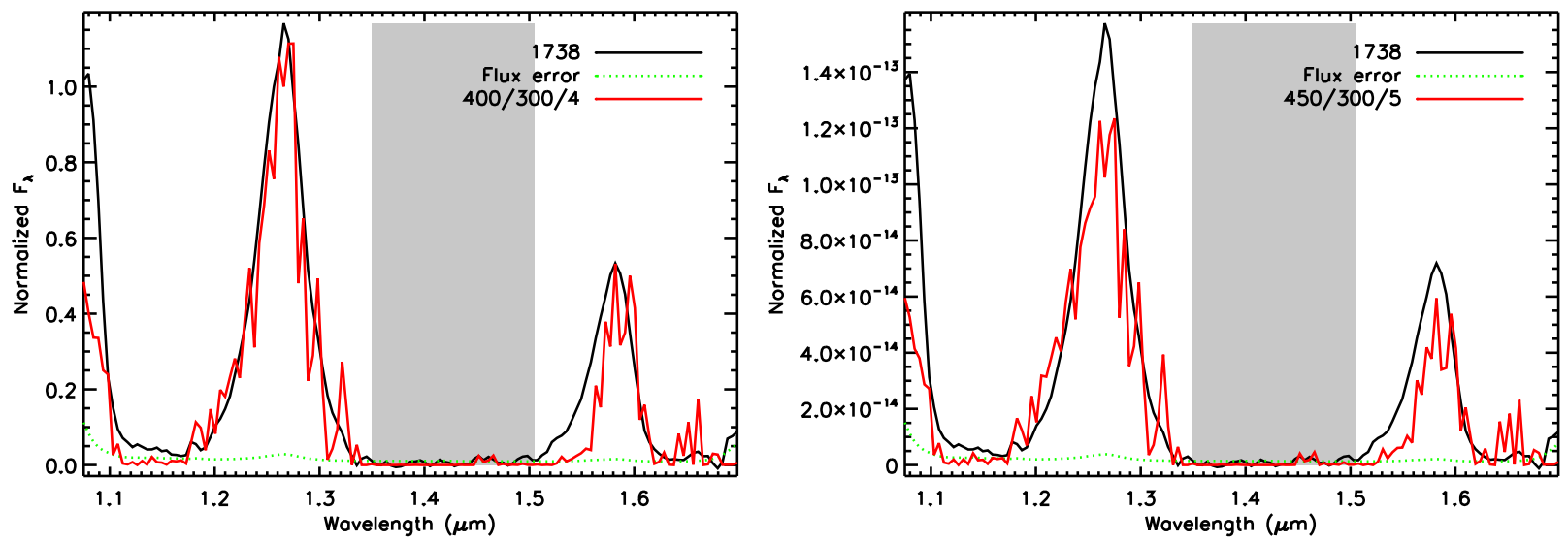

Morley et al. (2014) models.
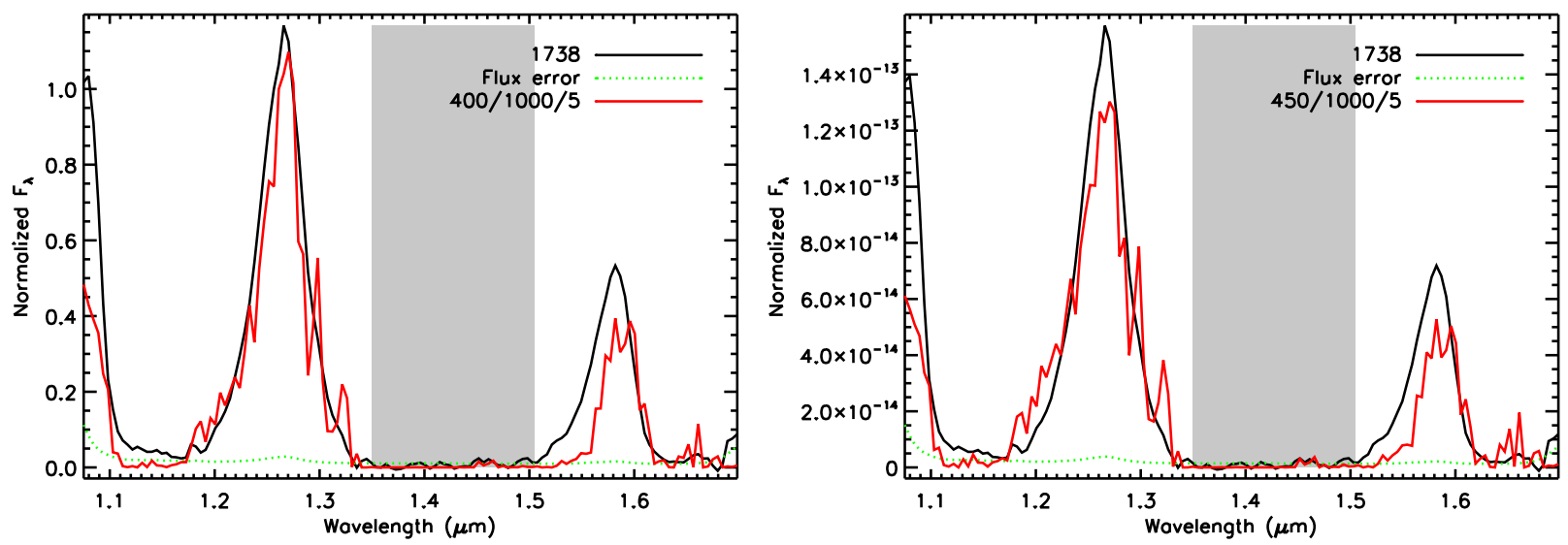

Figure 6. Same as Fig. 5 but for 1738 .

combination. The three $\mathrm{Y} 0$ objects studied here have absolute magnitudes that are consistent within the observational errors and we find mean absolute magnitudes for various passbands. A comparison to other parallax determinations shows our values to be consistent with those of Beichman et al. (2014) but indicates that the values in
Dupuy \& Kraus (2013) are underestimated. While Dupuy \& Kraus (2013) published only relative parallaxes, the observed difference is too large to be due to a required correction to an absolute value.

This difference must lie in the reduction procedures or in some systematic bias in the observational material. Parallaxes of objects 
Tremblin et al. (2015) models.
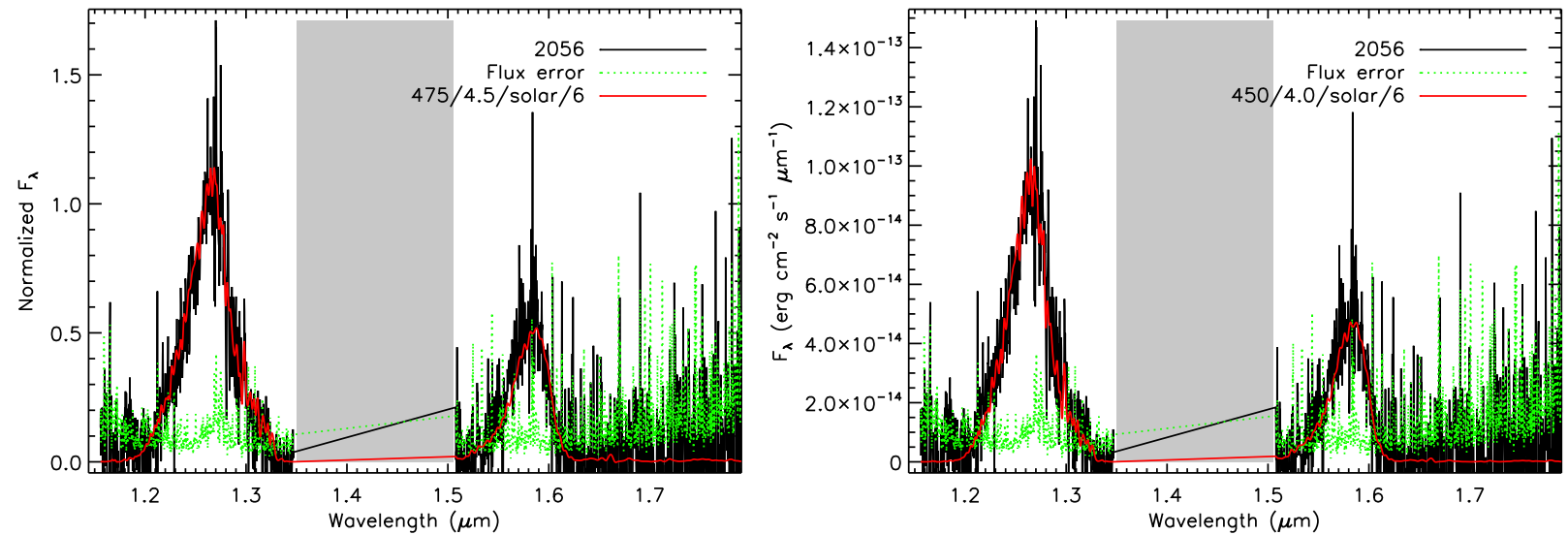

Morley et al. (2012) models.
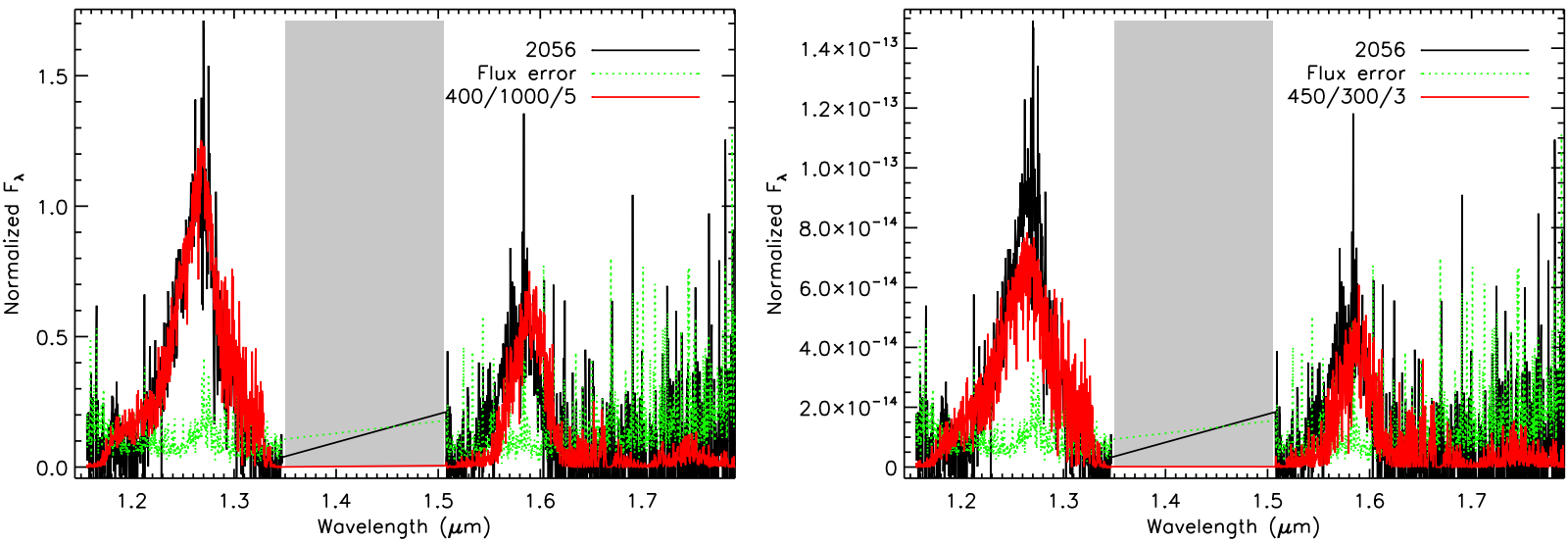

Morley et al. (2014) models.
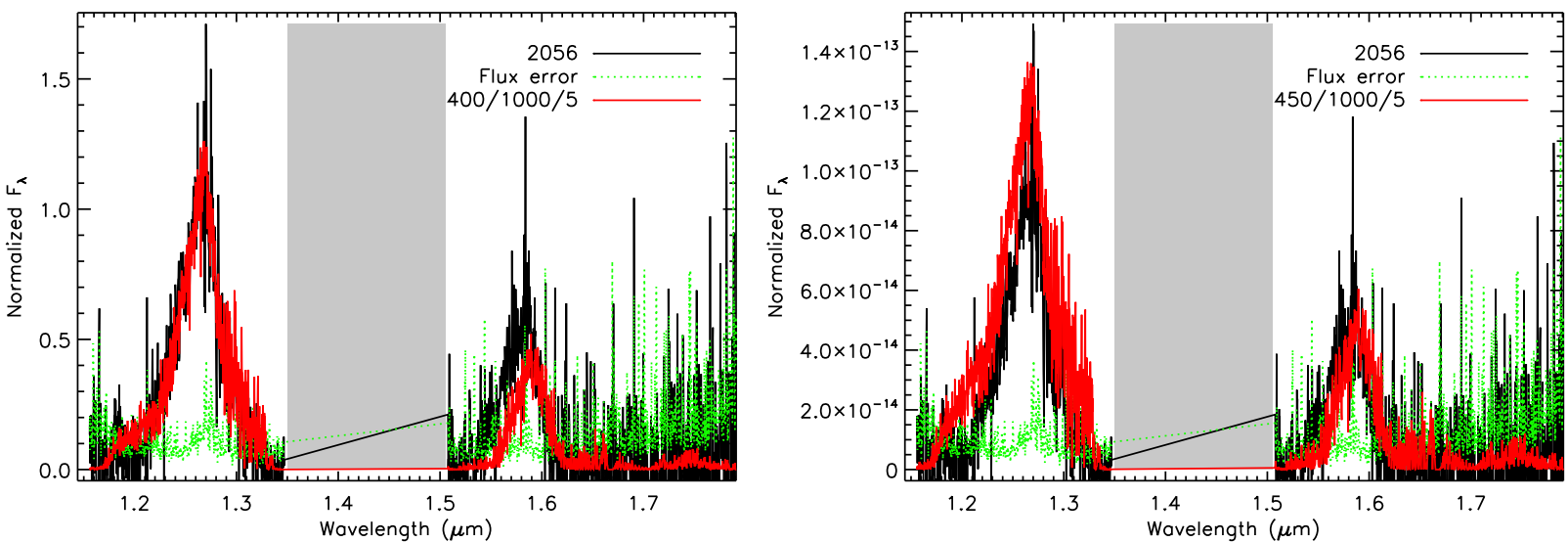

Figure 7. Same as Fig. 5 but for 2056.

this faint will remain the domain of relative small-field programs for the near future but with wealth of objects being published by Gaia a gold standard will be produced which will allow small-field programs to calibrate instruments and refine procedures to the submas level. Also, while Gaia will not observe directly any objects cooler than late $\mathrm{T}$ dwarfs it will indirectly detect cool objects in binary systems that will serve as direct comparisons in absolute magnitude space to those measured with small-field programs. The ability to detect and remove systematic errors from the observations and reductions along with a direct comparison sample will lead to 
more robust and consistent small-field results that will only aid the astrophysical interpretation.

As shown in Section 3, our multi-epoch observations sample the near-infrared brightness evolution of the three targets over a baseline of about $4 \mathrm{yr}$. Simple linear fits to the $J$ measurements show small and marginally statistically significant long-term slopes in two of our targets and indicate a possible slope in the third target (Table 3). Although the existence of long-term monotonic variations is tentative, this possibility is intriguing and in the following we briefly explore their possible nature.

Our measurements represent the first long-term precision photometry of Y dwarfs and, as such, the first probes of long-term atmospheric evolution in these objects. Recent precision nearinfrared (Spitzer) studies of $\mathrm{Y}$ dwarfs detected high-amplitude ( $\sim 3$ percent to 15 percent) rotational modulations in three targets: WISEP J140518.40+553421.5 (Cushing et al. 2016), WISE J173835.52+273258.9 (Leggett et al. 2016) and WISE J085510.83071442.5 (Esplin et al. 2016). These modulations are periodic on the $6 \mathrm{~h}$ time-scales and consistent with rotational modulation that are found to be common in $\mathrm{L}, \mathrm{L} / \mathrm{T}$ and T-type brown dwarfs by extensive and sensitive space-based surveys (Buenzli et al. 2014; Metchev et al. 2015). Detailed analysis of photometric variability in L/T dwarfs indicated variations of cloud properties (e.g. Artigau et al. 2009; Radigan et al. 2012), which was verified as correlated temperature and cloud thickness variations by high-precision timeresolved spectroscopy (Apai et al. 2013). In contrast, for mid-L-type brown dwarfs modulations in the condensate cloud properties failed to reproduce the observed grey variations and indicated a high-level haze layer (Yang et al. 2016). The observed modulations in Y dwarfs are probably emerging due to the rotational modulations introduced by heterogeneous $\mathrm{KCl}$ and $\mathrm{Na}_{2} \mathrm{~S}$ clouds (Leggett et al. 2016).

Unlike the above studies, our observations provide a sensitive probe of $\mathrm{Y}$ dwarfs over very long time-scales, i.e. over $10^{4}$ rotations. Our data hint on the possible existence of monotonic changes, which is not consistent with stochastic cloud evolution (occurring over dynamical time-scales, about a rotational period). Although the full interpretation of the variations is beyond the scope of this work, we embark here on a speculative discussion of the importance of the photometric variations. The changes, if real, are probably occurring due to a long-term monotonic evolution of the clouds, driven by a process that acts on a time-scale much longer than the dynamical time-scales. We note that chemical disequilibrium could drive such slow changes if the kinetic time-scales for one or more important processes are very long. An example of such slow, chemical disequilibrium-driven cloud evolution is given by the spectacular 2012 Saturn Storm (Sromovsky, Baines \& Fry 2013). During this event, water vapor rich air was dredged up from the deep interior of Saturn to its upper, cold and very dry atmosphere. Water and other volatiles froze out in the upper atmosphere and formed clouds that were optically thick at optical and infrared wavelengths. Over the course of the following six months the clouds encompassed Saturn's Northern hemisphere and eventually dispersed, with ice crystals likely settling to the deeper interior, leaving the upper atmosphere dry again. We speculate here that qualitatively similar events may drive long time-scale monotonic brightness evolution in Y dwarfs.

We have compared the combination of spectra and parallaxes to the atmospheric models of Tremblin et al. (2015) and Morley et al. (2012, 2014). We find that the best physical parameters are consistent between the three objects with solar metallicity, temperatures between 450 and $475 \mathrm{~K}$, and a $\log g$ of 4.0-4.5. A general consideration that arises from the model fitting is that, if we do not employ the measured parallax, the models alone would lead to overestimated $\log g$, i.e. we would essentially be overestimating the mass of our targets. We note that for Tremblin et al. (2015) all best-fitting models are non-equilibrium models, stressing the importance of mixing in such low-temperature atmospheres. All Tremblin et al. (2015) best-fitting models are solar-metallicity models; however, given our coarse metallicity grid, this result is less conclusive. All Y dwarfs have best-fitting $\log g \geq 4.0$, suggestive of 'old', evolved objects (age $>1 \mathrm{Gyr}$ ), in agreement with their kinematics. If we compare the parameters derived here with those presented in Cushing et al. (2011), we note that we either get a significantly higher $T_{\text {eff }}(\sim 100$ $150 \mathrm{~K}$ higher, for 1738 and 2056) or a significantly higher $\log g$ (0.75 dex, for 0410). While comparing the results from different model grids is a dangerous exercise (given the disparate underlying assumptions, the different parameter space covered and the heterogeneous steps of the grid), it is however remarkable to see such a large discrepancy in the predicted atmospheric parameters. Finally, we stress that this analysis is based on near-infrared spectra only, while Y dwarfs emit most of their energy at longer wavelengths. Any systematic issue with models in the near-infrared range would therefore lead to incorrect atmospheric parameters.

\section{ACKNOWLEDGEMENTS}

We thank the anonymous referee for comments that improved the clarity of this contribution; Isabelle Baraffe and Gilles Chabrier for useful discussions on the model aspects addressed here; Luca Rizzi, Tom Kerr, Watson Varricatt and Andy Adamson for scheduling help; and Mike Cushing who promptly provided the spectra of these three targets. This work has made use of the Cambridge Astronomy Survey Unit software and the WFCAM Science Archive thanks to Mike Irwin and Mike Read for their support. The United Kingdom Infrared Telescope was operated by the Joint Astronomy Centre on behalf of the Science and Technology Facilities Council of the UK, and it is currently operated by the University of Arizona. RLS's research was supported by the 2015 Henri Chrétien International Research Grant administered by the American Astronomical Society and a Visiting Professorship with the Leverhulme Trust (VP1-2015-063). SKL's research is supported by the Gemini Observatory, which is operated by the Association of Universities for Research in Astronomy, Inc., on behalf of the international Gemini partnership of Argentina, Australia, Brazil, Canada, Chile and the United States of America. FM/HRAJ/DJP acknowledges the support from UK's Science and Technology Facilities Council grant number ST/M001008/1. The collaboration was supported by the Marie Curie 7th European Community Framework Programme grant no. 247593 Interpretation and Parametrisation of Extremely Red COOL dwarfs (IPERCOOL) International Research Staff Exchange Scheme.

\section{REFERENCES}

Apai D., Radigan J., Buenzli E., Burrows A., Reid I. N., Jayawardhana R., 2013, ApJ, 768, 121

Artigau É., Bouchard S., Doyon R., Lafrenière D., 2009, ApJ, 701, 1534

Beichman C., Gelino C. R., Kirkpatrick J. D., Cushing M. C., DodsonRobinson S., Marley M. S., Morley C. V., Wright E. L., 2014, ApJ, 783, 68

Buenzli E., Apai D., Radigan J., Reid I. N., Flateau D., 2014, ApJ, 782, 77 Cushing M. C. et al., 2011, ApJ, 743, 50

Cushing M. C. et al., 2016, ApJ, 823, 152

Dupuy T. J., Kraus A. L., 2013, Science, 341, 1492

Dye S. et al., 2006, MNRAS, 372, 1227 
Esplin T. L., Luhman K. L., Cushing M. C., Hardegree-Ullman K. K., Trucks J. L., Burgasser A. J., Schneider A. C., 2016, ApJ, 832, 58

Faherty J. K., Burgasser A. J., Cruz K. L., Shara M. M., Walter F. M., Gelino C. R., 2009, AJ, 137, 1

Gagné J., Lafrenière D., Doyon R., Malo L., Artigau É., 2014, ApJ, 783, 121

Hodgkin S. T., Irwin M. J., Hewett P. C., Warren S. J., 2009, MNRAS, 394, 675

Kirkpatrick J. D. et al., 2011, ApJS, 197, 19

Kirkpatrick J. D. et al., 2012, ApJ, 753, 156

Leggett S. K., Morley C. V., Marley M. S., Saumon D., Fortney J. J., Visscher C., 2013, ApJ, 763, 130

Leggett S. K., Morley C. V., Marley M. S., Saumon D., 2015, ApJ, 799, 37

Leggett S. K. et al., 2016, ApJ, 830, 141

Lodieu N., Béjar V. J. S., Rebolo R., 2013, A\&A, 550, L2

Mainzer A. et al., 2011, ApJ, 726, 30

Malo L., Doyon R., Lafrenière D., Artigau É., Gagné J., Baron F., Riedel A., 2013, ApJ, 762, 88

Marocco F. et al., 2010, A\&A, 524, A38

Marsh K. A., Wright E. L., Kirkpatrick J. D., Gelino C. R., Cushing M. C., Griffith R. L., Skrutskie M. F., Eisenhardt P. R., 2013, ApJ, 762, 119

Mendez R. A., van Altena W. F., 1996, AJ, 112, 655

Metchev S. A. et al., 2015, ApJ, 799, 154
Morley C. V., Fortney J. J., Marley M. S., Visscher C., Saumon D., Leggett S. K., 2012, ApJ, 756, 172

Morley C. V., Marley M. S., Fortney J. J., Lupu R., Saumon D., Greene T., Lodders K., 2014, ApJ, 787, 78

Radigan J., Jayawardhana R., Lafrenière D., Artigau É., Marley M., Saumon D., 2012, ApJ, 750, 105

Saumon D., Marley M. S., 2008, ApJ, 689, 1327

Schneider A. C. et al., 2015, ApJ, 804, 92

Skrutskie M. F. et al., 2006, AJ, 131, 1163

Smart R. L. et al., 2010, A\&A, 511, A30

Sromovsky L. A., Baines K. H., Fry P. M., 2013, Icarus, 226, 402

Tinney C. G., Faherty J. K., Kirkpatrick J. D., Cushing M., Morley C. V., Wright E. L., 2014, ApJ, 796, 39

Tremblin P., Amundsen D. S., Mourier P., Baraffe I., Chabrier G., Drummond B., Homeier D., Venot O., 2015, ApJ, 804, L17

Warren S. J. et al., 2007, MNRAS, 375, 213

Wright E. L. et al., 2010, AJ, 140, 1868

Yang H. et al., 2016, ApJ, 826, 8

Yurchenko S. N., Tennyson J., 2014, MNRAS, 440, 1649

Yurchenko S. N., Barber R. J., Tennyson J., 2011, MNRAS, 413, 1828

This paper has been typeset from a $\mathrm{T}_{\mathrm{E}} \mathrm{X} / \mathrm{LAT}_{\mathrm{E}} \mathrm{X}$ file prepared by the author. 